\title{
IV. Red List of Vascular Plants of the Wadden Sea Area*
}

\author{
CONTRIBUTORS: \\ Denmark: P. Wind \\ Germany: M. van der Ende, E. Garve, A. Schacherer \\ The Netherlands: J. B. M. Thissen “
}

\section{INTRODUCTION}

In the aquatic part of the Wadden Sea area, there are only very few vascular plants, mainly Zostera marina and $Z$. noltii, but floristically the Wadden Sea islands are among the most important areas of northwestern Europe, especially for plants of coastal dunes and salt marshes (Mennema \& Weeda, 1983). However, according to Raabe (1981), the Schleswig-Holstein part is relatively poor in this respect.

One has to bear in mind that the number of vascular plants of some Wadden Sea islands has increased a lot in this century: e.g. for Vlieland, Terschelling, Ameland and Schiermonnikoog, the flora list comprised 60-90\% more species in 1986 than in 1870 (Westhoff \& van Oosten, 1991). About one century ago, the dunes were in a very poor state because of intensive exploitation (Westhoff, 1985).

\section{Delimitation of the area and selection of species}

At the Trilateral Governmental Conference in Esbjerg, it was decided to work out a Red List of the species which can be found in and which are typical for typical coastal habitats only. The vascular plants of the Wadden Sea itself (aquatic and intertidal part), the salt marshes and the dunes, excluding plantations, have been taken into consideration. So, for example, the flowering plants of arable land have been deliberately excluded from the Red List. (The weed flora of the Wadden Sea islands used to be very rich [Westhoff \& van Oosten, 1991].)

Further, the list has been restricted to indigenous or naturalized species. As a guideline, the lists of Prins et al. (1983 a, b) have been used. The apomictic-species of the genera Rubus and Taraxacum have not been assessed, because of lack of information on

\footnotetext{
- This list forms part of the Report on the RED LISTS OF BIOTOPES, FLORA AND FAUNA OF THE TRILATERAL WADDEN SEA AREA. For basic information concerning, for example, function of these lists, species taken into account, structure of the lists and abbreviations used, see also the general introduction to the Red Lists.

* Author of the introductory text.
} 
their distribution and status. Subspecies are only mentioned if this adds any value, e.g. if the specific subspecies is threatened, whereas the species as such is not.

The taxonomy and the scientific names follow the standard list for plants in Germany (Zentralstelle für die floristische Kartierung, 1993).

\section{Data source}

Denmark: As in the case of the other species groups, the Danish Wadden Sea Red List of vascular plants is an updated version of the Danish national Red List (Asbirk \& Søgaard, 1991).

Germany: The status of the vascular plants of Niedersachsen has been based, to a large extent, on the Red List of that region (Garve, 1993). For Schleswig-Holstein, however, the status categories of the Red List (Mierwald \& Beller, 1990) had to be adapted to the situation in the Wadden Sea part.

The Netherlands: The status of the species has been determined with the aid of the atlases by Mennema et al. $(1980,1985)$ and van der Meijden et al. (1989), which give the distribution in the two periods before and after 1950. This information was updated from some new publications, mainly Westhoff \& van Oosten (1991) and Weeda et al. (19851994).

\section{Threats}

In 1956, Westhoff pointed out that the loss of plant species in the coastal dunes has in general been relatively slight. At that time, the main threats were extraction of drinking water, drainage of adjacent agricultural fields, which cause the desiccation of humid and wet dune slacks, and afforestation, which causes desiccation too and even directly the destruction of natural habitats. Nowadays, one has to take more threats into account, namely atmospheric deposition of nitrogen (Bellemakers et al., 1993; Veer et al., 1993), recreation (van Zoest, 1994), regulation of moving sand and coastal defense (Janssen, 1994).

Desiccation and atmospheric deposition of nitrogen cause the dominance of a few grasses and of the invasive neophyte moss Campylopus introflexus. Recreation concentrates on the beach and disturbs typical beach plants, like the three Atriplex species of the Red List. Pioneer plants are threatened by fixation of moving sand. The flora of the outer dunes is threatened by regression of the coastline, but also to some extent by the measures which are taken to counteract this process. A specific effect of coastal defense is the loss of brackish waters. Several plants of gradients between fresh and salt water are disappearing. A lot of salt marshes and their characteristic plants disappeared locally because of embanking and reclamation.

In 1932 the sublittoral Zostera marina populations perished because of the "wasting disease". After 1965, also Zostera noltii began to decline in the Dutch part because of increasing pollution (den Hartog \& Polderman, 1975). The relevant effects of eutrophication are turbidity and high densities of microalgae and lugworms (Arenicola marina L.) (Philippart \& Dijkema, 1995). Since about 1972 Zostera noltii has been recovering in the Dutch part. In the German Wadden Sea mass development of macroalgae probably caused a recent decline of seagrass (Reise et al, 1994). Seagrasses are also threatened by fishery of shellfish (de Jonge \& de Jong, 1992). 
Regulation of human disturbance is not always beneficial to the flora. Some rare species benefit from a certain, slight, amount of eutrophication or treading, e.g. the Red List species Alyssum alyssoides, Anacamptis pyramidalis, Asparagus officinalis ssp. prostratus and Dactylorhiza maculata. On some salt marshes, especially in Schleswig-Holstein, grazing pressure restricts plant diversity (Stock, 1993). Low intensity grazing, however, can be beneficial to the flora of salt marshes (Scherfose, 1993) and dunes (Westhoff, 1985).

\section{Summary}

In the Wadden Sea area, a total of 248 (sub)species of vascular plants are threatened in at least one subregion. Of these, 216 (sub)species are threatened in the entire area and are therefore placed on the trilateral Red List. 17 (sub)species of the listed vascular plants are (probably) extinct in the entire Wadden Sea area. The status of 47 (sub)species of vascular plants is (probably) critical; 61 (sub)species are (probably) endangered; the status of 65 (sub)species is (probably) vulnerable and that of 26 (sub)species susceptible.

\section{REFERENCES}

Asbirk, S. \& S. Søgaard (Eds), 1991. "Rødliste 90“. - Saerligt beskyttelseskræevende planter og dyr i Danmark. (Red Data book of threatened plants and animals in Denmark - with English summary) - Miljøministeriet, Skov- og Naturstyrelsen, København, 222 pp.

Bellemakers, M. J. S., Maessen, M., Cals, M. J. R. \& J. G. M. Roelofs, 1993. Effectgerichte maatregelen tegen verzuring en eutrofiëring van oppervlaktewateren. Katholieke Universiteit, Nijmegen, 147 pp.

Garve, E., 1993. Rote Liste der gefährdeten Farn- und Blütenpflanzen in Niedersachsen und Bremen. 4. Fassung vom 1.1.1993. - Infn. Naturschutz Nieders. 13, 1-37.

Gravesen, P., 1983. Foreløbig oversigt over botaniske lokaliteter. 4. Sønderjyllands Amt. - Miljøministeriet, Fredningsstyrelsen, København, $158 \mathrm{pp}$.

Hansen, K. (red.), 1981. Dansk feltflora.- Gyldendal, København, 757 pp.

Hartog, C. den \& P. J. G. Polderman, 1975. Changes in the seagrass populations of the Dutch Waddenzee. - Aquat. Bot, 1, 141-147.

Janssen, M., 1994. Dynamisch kustbeheer. - Duin 17(2), 17-19.

Jonge, V. N. de \& D. J. de Jong, 1992. Role of tide, light and fisheries in the decline of Zostera marina L. in the Dutch Wadden Sea. - Netherl. Inst. Sea Res. Publ. Ser. 20, 161-176.

Meijden, R. van der, Plate, C. L. \& E. J. Weeda, 1989. Atlas van de Nederlandse flora. 3: Minder zeldzame en algemene soorten. Rijksherbarium, Leiden, $264 \mathrm{pp}$.

Mennema, J., Quéne-Boterenbrood, A. J. \& C. L. Plate (Eds), 1980. Atlas van de Nederlandse flora. 1: Uitgestorven en zeer zeldzame planten. Kosmos, Amsterdam, $226 \mathrm{pp}$.

Mennema, J., Quéne-Boterenbrood, A. J. \& C. L. Plate (Eds), 1985. Atlas van de Nederlandse flora. 2: Zeldzame en vrij zeldzame planten. Bohn, Scheltema \& Holkema, Utrecht, $349 \mathrm{pp}$.

Mennema, J. \& E. J. Weeda, 1983. Vascular plants. In: Ecology of the Wadden Sea. Ed. by W. J. Wolff. Balkema, Rotterdam, 3, 9/38-9/50.

Mierwald, U. \& J. Beller, 1990. Rote Liste der Farn- und Blütenpflanzen Schleswig-Holsteins. 3. Fassung. Landesamt für Naturschutz and Landschaftspflege, Kiel, 64 pp.

Pedersen, A., 1983. The vegetation of the Wadden Sea islands of Denmark and Schleswig-Holstein. In: Ecology of the Wadden Sea. Ed. by W. J. Wolff. Balkema, Rotterdam, 3, 9/137-9/165.

Philippart, C. J. M. \& K. S. Dijkema, 1995. Wax and wane of Zostera noltii L. in the Dutch Wadden Sea. - Aquat. Bot. 49, 255-268.

Prins, D., Kuhbier, H., Pedersen, A., Mennema, J. \& A. M. Kooijman, 1983 a. Main list with indigenous or naturalized phanerogams and pteridophytes of the Wadden Sea area. In: Ecology of the Wadden Sea. Ed. by W. J. Wolff. Balkema, Rotterdam, 3, 9/323-9/344. 
Prins, D., Kuhbier, H., Pedersen, A., Mennema, J. \& A. M. Kooijman, 1983 b. Separate list with indigenous or naturalized plants of smaller islands and "high-sands”. In: Ecology of the Wadden Sea. Ed. by W. J. Wolff. Balkema, Rotterdam, 3, 9/345-9/346.

Raabe, E.W., 1981. Über das Vorland der östlichen Nordseeküste. - Mitt. ArbGem. Geobotanik in Schleswig-Holstein und Hamburg 31,1-118.

Reise, K., Kolbe, K. \& V. de Jong, 1994. Makroalgen und Seegrasbestände im Wattenmeer. In: Warnsignale aus dem Wattenmeer. Ed. by J. L. Lozan, E. Rachor, K. Reise, H. von Westernhagen \& W. Lenz. Blackwell, Berlin, 90-100.

Scherfose, V., 1993. Zum Einfluß der Beweidung auf das Gefäßpflanzen-Artengefüge von Salz- und Brackmarschen. - Zeitschr. Ökol. Naturschutz 2, 201-211.

Stock, M., 1993. Salt marshes in Schleswig-Holstein: from a green towards a natural succession. Wadden Sea Newsletter 1993-1, 11-14.

Veer, M., Meulen, F. van der, Heil, G. W. \& A. M. Kooijman, 1993. Ecotoopbeheer in droge duinen: sturende processen en limiterende factoren. In: Effectgerichte maatregelen tegen verzuring en eutrofiëring in natuurterreinen. Ed. by M. Cals, Graaf, M. de \& J. Roelofs. Katholieke Universiteit, Nijmegen, 147-170.

Westhoff, V., 1956. De verarming van flora en vegetatie. In: Vijftig jaar natuurbescherming in $\mathrm{Ne}$ derland. Natuurmonumenten, Amsterdam, 151-186.

Weeda, E. J., Westra, R., Westra, C. \& T. Westra, 1985. Nederlandse oecologische flora 1. IVN, Amsterdam, $304 \mathrm{pp}$.

Weeda, E. J., Westra, R., Westra, C. \& T. Westra, 1987. Nederlandse oecologische flora 2. IVN, Amsterdam, $304 \mathrm{pp}$.

Weeda, E. J., Westra, R., Westra, C. \& T. Westra, 1988. Nederlandse oecologische flora 3. IVN, Amsterdam, $302 \mathrm{pp}$.

Weeda, E. J., Westra, R., Westra, C. \& T. Westra, 1991. Nederlandse oecologische flora 4. IVN, Amsterdam, $317 \mathrm{pp}$.

Weeda, E. J., Westra, R., Westra, C. \& T. Westra, 1994. Nederlandse oecologische flora 5. IVN, Amsterdam, $400 \mathrm{pp}$.

Westhoff, V., 1985. Nature management in coastal areas of Western Europe. - Vegetation 62, 523532.

Westhoff, V. \& M.F. van Oosten, 1991. Plantengroei van de Waddeneilanden. KNNV, Utrecht, 417 pp.

Wind, P., 1994 a. Oversigt over botaniske lokaliteter. Bind 10. Ribe Amt.- Miljøministeriet, Fredningsstyrelsen, Skov- og Naturstyrelsen, København, 176 pp.

Wind, P., 1994 b. Oversigt over botaniske lokaliteter. Status og forvaltningsbehov.- Miljøministeriet, Fredningsstyrelsen, Skov- og Naturstyrelsen, København, Vol. 1, 68 pp; Vol. 2, 94 pp.

Zentralstelle für die floristische Kartierung der Bundesrepublik Deutschland-Nord (Hrsg.), 1993. Standardliste der Farn- und Blütenpflanzen der Bundesrepublik. - Floristische Rundbr. Beih. 3, $480 \mathrm{pp}$.

Zoest, J. van, 1994. Effecten van openluchtrecreatie op natuurwaarden van de Waddeneilanden. Landelijke Vereniging tot Behoud van de Waddenzee, Harlingen, $74 \mathrm{pp}$.

\section{RED LIST OF VASCULAR PLANTS OF THE WADDEN SEA AREA*}

\section{EX - Extinct:}

Ajuga pyramidalis

(?) Alyssum alyssoides

Anacamptis pyramidalis

Carex dioica

Carex hostiana

Chenopodium botryodes
Erica cinerea

(?) Filago lutescens

Genista germanica

Hieracium vulgatum

(?) Hypericum humifusum

Illecebrum verticillatum

(?) Marrubium vulgare

* Question-marks indicate that in one of the subregions the status of threat is uncertain. 
(?) Ranunculus hederaceus

Ranunculus tripartitus

Subularia aquatica

Zannichellia palustris ssp. polycarpa

CR - Critical:

Arnica montana

Artemisia campestris

Baldellia ranunculoides

Bassia hirsuta

Blysmus compressus

Callitriche brutia

Calystegia soldanella

Carex caryophyllea

Carex ericetorum

Carex pulicaris

Carex punctata

Catabrosa aquatica

Cicendia filiformis

Cochlearia officinalis

Crambe maritima

Cuscuta epithymum

Deschampsia setacea

Dianthus carthusianorum

Dianthus deltoides

Eleocharis acicularis

Equisetum variegatum

Eriophorum vaginatum

Euphorbia paralias

Filago vulgaris

Gentianella campestris ssp.baltica

Gnaphalium luteo-album

Hammarbya paludosa

Herminium monorchis

Hordeum marinum

Hottonia palustris

Huperzia selago

Juncus capitatus

Juniperus communis

Orchis morio

Pilularia globulifera

Pinguicula vulgaris

(?) Rhynchospora alba

Rhynchospora fusca

Sagina subulata

Schoenoplectus pungens
Silene nutans

Sparganium natans

Stellaria aquatica

(?) Thalictrum flavum

Trichophorum cespitosum

Tuberaria guttata

Utricularia minor

EN - Endangered:

Acinos arvensis =Satureja acinos

Alopecurus bulbosus

Anagallis minima $=$ Centunculus minimus

Antennaria dioica

Apium graveolens

Apium inundatum

Atriplex glabriuscula

Atriplex laciniata

Botrychium lunaria

Bupleurum tenuissimum

Carex trinervis

Carex viridula

$=$ C.oederi

$=$ C. serotina ssp. serotina

Carlina vulgaris

Cirsium dissectum

Cotula coronopifolia

Dactylorhiza incarnata

Drosera intermedia

Epipactis palustris

Erica scoparia

Euphrasia micrantha

Galium sterneri

Gentiana pneumonanthe

Gentianella uliginosa

Gymnadenia conopsea

Halimione pedunculata

Helictotrichon pubescens

Hypericum pulchrum

Hypochoeris glabra

Isolepis fluitans

Juncus pygmaeus

Lathyrus linifolius

Limosella aquatica

Liparis loeselii

Listera cordata

Littorella uniflora 
(?) Lysimachia thyrsiflora

(?) Myosurus minimus

Myriophyllum alterniflorum

Narthecium ossifragum

Odontites litoralis

Oenanthe lachenalii

Pedicularis palustris

Pedicularis sylvatica

Platanthera bifolia

Polygala serpyllifolia

Polygonum oxyspermum ssp. raii

Potamogeton gramineus

Potamogeton perfoliatus

Pyrola rotundifolia

Ruppia cirrhosa

Ruppia maritima

Schoenoplectus triqueter

Schoenoplectus $X$ carinatus

Schoenus nigricans

Scorzonera humilis

Scutellaria minor

Senecio erucifolius

(?) Stachys arvensis

Ulex europaeus

Vaccinium vitis-idaea

Vulpia bromoides

\section{VU - Vulnerable:}

Agrostis vinealis $=$ A. stricta ssp. stricta

Anagallis tenella

(?) Anthyllis vulneraria ssp. maritima

(?) Artemisia absinthium

Asparagus officinalis ssp. prostratus

(?) Atriplex longipes

Blysmus rufus

Briza media

Caltha palustris

Carex distans

Carex extensa

Carex vesicaria

Carex viridula var. pulchella

$=$ C.oederi ssp. oederi

=C.oederi ssp. pulchella

=C.scandinavica

$=$ C. serotina spp. pulchella

Centaurium erythraea
Crepis tectorum

Dactylorhiza maculata

Dactylorhiza majalis

Dactylorhiza praetermissa

Eleocharis multicaulis

Eleocharis quinqueflora

Eryngium maritimum

Euphrasia stricta

Filago minima

(?) Galeopsis speciosa

Halimione portulacoides $=$ Atriplex portu-

lacoides

Hierochloe odorata

Inula britannica

Juncus anceps

Juncus balticus

Juncus inflexus

Lathyrus maritimus $=L$. japonicus

Lepidium campestre

Linum catharticum

Lycopodiella inundata

Lycopodium clavatum

Menyanthes trifoliata

Ophioglossum vulgatum

Osmunda regalis

Parapholis strigosa

Parnassia palustris

Peplis portula

Polygala vulgaris

Potamogeton polygonifolius

Potamogeton trichoides

Potentilla argentea

Pyrola minor

Radiola linoides

Ranunculus baudotii

Rhinanthus angustifolius

(?) Rhinanthus angustifolius ssp. halophiIus

Rhinanthus minor

Rosa pimpinellifolia

Sagina nodosa

Samolus valerandi

Scleranthus perennis

Silene otites

Taraxacum celticum agg.

Taraxacum palustre agg. 
Thalictrum minus

Torilis nodosa

Triglochin palustre

Utricularia australis

Vaccinium myrtillus

Zostera marina

Zostera noltii

\section{SU - Susceptible:}

Ajuga reptans

Alisma lanceolatum

Apium nodiflorum

Arabis hirsuta

Berberis vulgaris

Carex hartmanii

Carex lasiocarpa

Carex remota

(?) Cerastium pumilum
(?) Cicuta virosa

Crithmum maritimum

Deschampsia wibeliana

Desmazeria marina

Galeopsis segetum

(?) Juncus bulbosus ssp. kochii

Lactuca tatarica

Lithospermum officinale

Polygonatum odoratum

Potamogeton coloratus

Ranunculus auricomus

Sedum album

Smyrnium olusatrum

Spergula morisonii

Thymus pulegioides

Trifolium striatum

Xanthium albinum ssp. albinum

\section{LIST OF THREATENED VASCULAR PLANTS \\ OF THE WADDEN SEA AREA}

\begin{tabular}{|c|c|c|c|c|c|c|c|}
\hline & & \multirow{2}{*}{$\begin{array}{c}\text { Red List } \\
\text { (trilateral) }\end{array}$} & \multirow[t]{2}{*}{ Threats } & \multicolumn{4}{|c|}{$\begin{array}{l}\text { Status of threat in the subre- } \\
\text { gions of the Wadden Sea Area }\end{array}$} \\
\hline & & & & $\mathrm{NL}$ & Nds & $\mathrm{SH}$ & DK \\
\hline $\begin{array}{l}\text { Acinos arvensis (Lam.)Dandy } \\
=\text { Satureja acinos (L.) Scheele }\end{array}$ & $\begin{array}{l}\text { Kleine steentijm } \\
\text { Gemeiner Steinquendel } \\
\text { Voldtimian }\end{array}$ & EN & HAB, AGR & - & - & EN & - \\
\hline $\begin{array}{l}\text { Agrostis vinealis Schreb. } \\
=A . \text { stricta J.F.Gmelin ssp. stricta }\end{array}$ & $\begin{array}{l}\text { Zandstruisgras } \\
\text { Sand-Straußgras } \\
\text { Sand-Hvene }\end{array}$ & vu & $A G R, H A B$ & * & * & CR & - \\
\hline Ajuga pyramidalis L. & $\begin{array}{l}\text { Piramidezenegroen } \\
\text { Pyramiden-Günsel } \\
\text { Pyramide-Læbeløs }\end{array}$ & EX & & - & - & EX & - \\
\hline Ajuga reptans $\mathrm{L}$. & $\begin{array}{l}\text { Kruipend zenegroen } \\
\text { Kriech-Günsel } \\
\text { Krybende Læbeløs }\end{array}$ & su & & SU & - & - & - \\
\hline Alisma lanceolatum with. & $\begin{array}{l}\text { Slanke waterweegbree } \\
\text { Lanzett-Froschloffel } \\
\text { Lancet-Skeblad }\end{array}$ & su & & SU & - & - & - \\
\hline Alopecurus bulbosus Gouan & $\begin{array}{l}\text { Knolvossestaart } \\
\text { Knollen-Fuchsschwanz } \\
\text { Knold-Rævehale } \\
\end{array}$ & EN & WAT & EN & EN & - & - \\
\hline Alyssum alyssoides (L.) L.' & $\begin{array}{l}\text { Bleek schildzaad } \\
\text { Kelch-Steinkraut } \\
\text { Grådodder }\end{array}$ & EX? & $?$ & EX & EX & - & $(*)$ \\
\hline
\end{tabular}




\begin{tabular}{|c|c|c|c|c|c|c|c|}
\hline & & \multirow{2}{*}{$\begin{array}{c}\text { Red List } \\
\text { (trilateral) }\end{array}$} & \multirow[t]{2}{*}{ Threats } & \multicolumn{4}{|c|}{$\begin{array}{l}\text { Status of threat in the subre- } \\
\text { gions of the Wadden Sea Area }\end{array}$} \\
\hline & & & & NL & Nds & $\mathrm{SH}$ & DK \\
\hline $\begin{array}{l}\text { Anacamptis pyramidalis } \\
\text { (L.) Rich. }\end{array}$ & $\begin{array}{l}\text { Hondskruid } \\
\text { Pyramiden-Spitzorchis } \\
\text { Horndrager }\end{array}$ & EX & AGR & EX & - & - & - \\
\hline $\begin{array}{l}\text { Anagallis minima (L.) } \\
\text { E.H.L.Krause } \\
(=\text { Centuncutus minimus L.) }\end{array}$ & $\begin{array}{l}\text { Dwergbloem } \\
\text { Acker-Kleinling } \\
\text { Knudearve }\end{array}$ & EN & AGR, WAT & Vu & EN & $\mathrm{CR}$ & vu \\
\hline Anagallis tenella $(\mathrm{L}.) \mathrm{L}$. & $\begin{array}{l}\text { Teer guichelheil } \\
\text { Zarter Gauchheil } \\
\text { Spæd arve }\end{array}$ & Vu & WAT & VU & - & - & - \\
\hline Antennaria dioica (L.) Gaertn. & $\begin{array}{l}\text { Rozenkransje } \\
\text { Gemeines Katzenpfötchen } \\
\text { Almindelig Kattefod }\end{array}$ & EN & EUT & Vu & EX & $C R$ & * \\
\hline $\begin{array}{l}\text { Anthyllis vulneraria ssp. } \\
\text { maritima (Schweigg.) Corb. }\end{array}$ & $\begin{array}{l}\text { Wondklaver } \\
\text { Gemeiner Wundklee } \\
\text { Rundbælg }\end{array}$ & VU? & WAT & * & VU & - & $?$ \\
\hline Apium graveolens L. & \begin{tabular}{|l} 
Selderij \\
Echter Sellerie \\
Vild Selleri \\
\end{tabular} & EN & WAT, AGR & EN & EN & $\mathrm{CR}$ & - \\
\hline Apium inundatum (L.) Rchb.fil. & $\begin{array}{l}\text { Ondergedoken moeras- } \\
\text { scherm } \\
\text { Flutender Sellerie } \\
\text { Svømmende Sumpskærm }\end{array}$ & EN & $\begin{array}{l}\text { HAB, EUT, } \\
\text { AGR, WAT }\end{array}$ & Vu & EX & EN & SU \\
\hline Apium nodiflorum (L.) Lag. & $\begin{array}{l}\text { Groot moerasscherm } \\
\text { Knotenblütiger Scheiberich } \\
\text { Vand-Selleri }\end{array}$ & su & & SU & - & - & - \\
\hline Arabis hirsuta (L.) Scop. & \begin{tabular}{|l} 
Ruige scheefkelk \\
Rauhaarige Gänsekresse \\
Stivhăret Kalkkarse \\
\end{tabular} & su & & SU & - & - & - \\
\hline Armeria maritima (Mill.) Willd. & $\begin{array}{l}\text { Engels gras } \\
\text { Gemeine Grasnelke } \\
\text { Engelskgræs }\end{array}$ & * & AGR, WAT & VU & * & • & * \\
\hline Arnica montana L. & $\begin{array}{l}\text { Wolverlei } \\
\text { Arnika } \\
\text { Alm. Guldblomme } \\
\end{array}$ & CR & $\begin{array}{c}\text { EUT, AGR, } \\
\text { DIS }\end{array}$ & EX & EX & EN & su \\
\hline Artemisia absinthium L. ${ }^{\dagger}$ & $\begin{array}{l}\text { Absintalsem } \\
\text { Wermut } \\
\text { Have-Malurt }\end{array}$ & vU? & $?$ & $C R$ & * & $(*)$ & $(*)$ \\
\hline Artemisia campestris L. & $\begin{array}{l}\text { Averuit } \\
\text { Feld-Beifuß } \\
\text { Mark-Bynke }\end{array}$ & CR & AGR & su & EX & SU & * \\
\hline $\begin{array}{l}\text { Asparagus officinalis L. Ssp. } \\
\text { prostratus (Dum.) Corb. }\end{array}$ & $\begin{array}{l}\text { Liggende asperge } \\
\text { Spargel (ssp. prostratus) } \\
\text { Asparges (ssp. prostratus) }\end{array}$ & vu & AGR & EN & $*$ & - & - \\
\hline
\end{tabular}




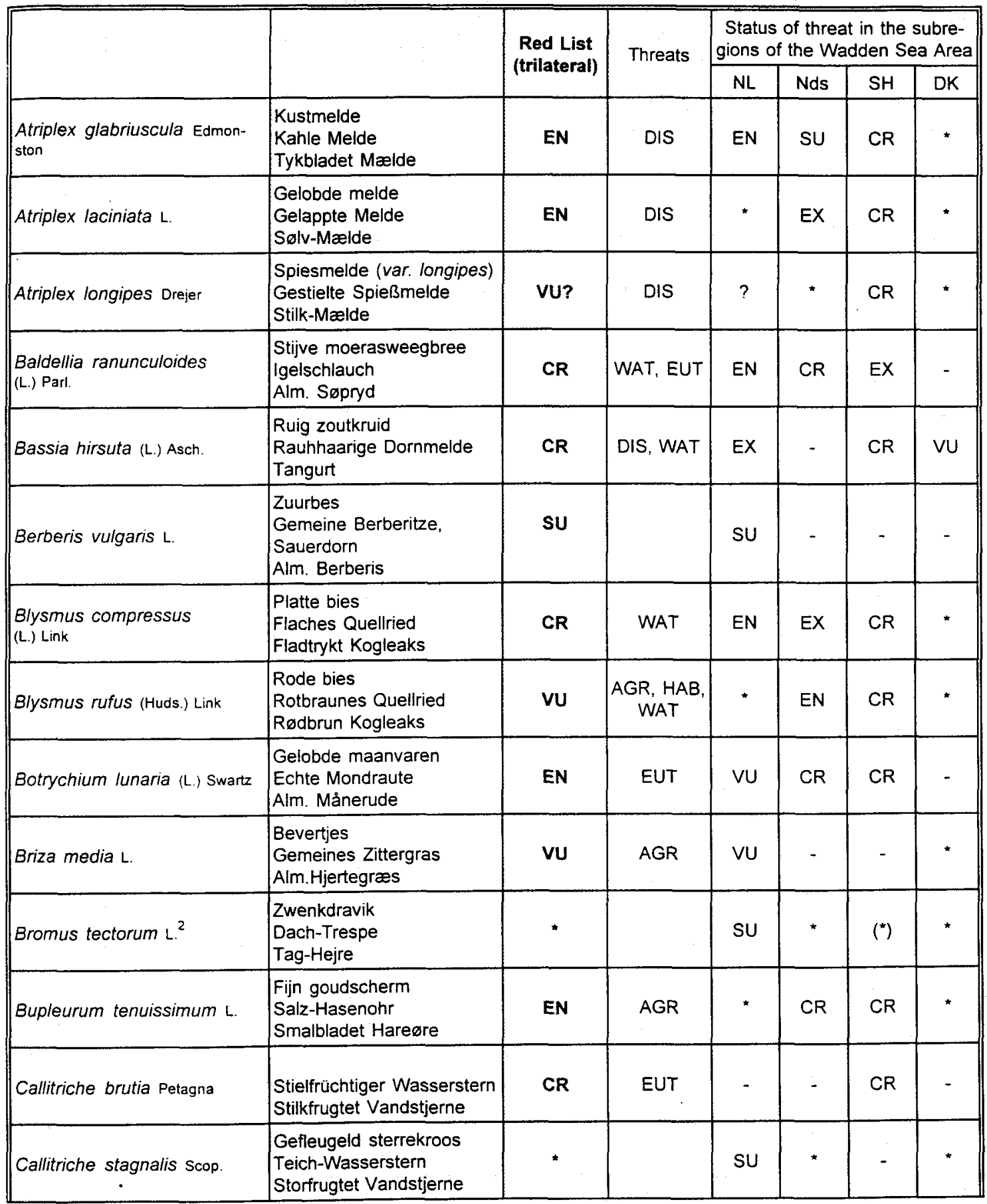




\begin{tabular}{|c|c|c|c|c|c|c|c|}
\hline & & \multirow{2}{*}{$\begin{array}{c}\text { Red List } \\
\text { (trilateral) }\end{array}$} & \multirow[t]{2}{*}{ Threats } & \multicolumn{4}{|c|}{$\begin{array}{l}\text { Status of threat in the subre- } \\
\text { gions of the Wadden Sea Area }\end{array}$} \\
\hline & & & & NL & Nds & $\mathrm{SH}$ & DK \\
\hline Caltha palustris $\mathrm{L}$. & $\begin{array}{l}\text { Dotterbloem } \\
\text { Sumpf-Dotterblume } \\
\text { Eng-Kabbeleje }\end{array}$ & vu & WAT & Vu & VU & VU & * \\
\hline $\begin{array}{l}\text { Calystegia soldanella } \\
\text { (L.) Roem. \& Schuit. }\end{array}$ & $\begin{array}{l}\text { Zeewinde } \\
\text { Strand-Winde } \\
\text { Strand-Snerle }\end{array}$ & CR & DIS & su & $\mathrm{CR}$ & $\mathrm{CR}$ & su \\
\hline Carex canescens L. & $\begin{array}{l}\text { Zompzegge } \\
\text { Grau-Segge } \\
\text { Grå Star }\end{array}$ & * & & SU & * & * & * \\
\hline Carex caryophyllea Lat. & $\begin{array}{l}\text { Voorjaarszegge } \\
\text { Frühlings-Segge } \\
\text { Vår-Star }\end{array}$ & CR & AGR & EX & - & EN & - \\
\hline Carex dioica L. & $\begin{array}{l}\text { Tweehuizige zegge } \\
\text { Zweihäusige Segge } \\
\text { Tvebo Star }\end{array}$ & EX & $\begin{array}{c}\text { EUT, WAT, } \\
\text { AGR }\end{array}$ & EX & EX & EX & - \\
\hline Carex distans L. & \begin{tabular}{|l} 
Zilte zegge \\
Entferntährige Segge \\
Fjernakset Star \\
\end{tabular} & VU & $A G R$ & • & VU & EN & * \\
\hline Carex ericetorum Pollich & $\begin{array}{l}\text { Heidezegge } \\
\text { Heide-Segge } \\
\text { Lyng-Star }\end{array}$ & CR & AGR, EUT & - & - & $\mathrm{CR}$ & - \\
\hline Carex extensa Good. & $\begin{array}{l}\text { Kwelderzegge } \\
\text { Strand-Segge } \\
\text { Udspilet Star }\end{array}$ & Vu & $A G R, H A B$ & * & su & EN & * \\
\hline Carex flacca Schreb. & \begin{tabular}{|l} 
Zeegroene zegge \\
Blaugrüne Segge \\
Blågrøn Star \\
\end{tabular} & * & AGR, WAT & * & VU & * & * \\
\hline Carex hartmanii Caj. & $\begin{array}{l}\text { Kleine Knotszegge } \\
\text { Hartmans Segge } \\
\text { Hartmans Star }\end{array}$ & su & & su & - & - & - \\
\hline Carex hostiana DC. & $\begin{array}{l}\text { Blonde zegge } \\
\text { Saum-Segge } \\
\text { Skede-Star }\end{array}$ & EX & WAT, AGR & EX & EX & EX & - \\
\hline Carex lasiocarpa Ehrh. & $\begin{array}{l}\text { Draadzegge } \\
\text { Faden-Segge } \\
\text { Tråd-Star } \\
\end{array}$ & SU & & su & - & - & - \\
\hline Carex pulicaris L. & \begin{tabular}{|l} 
Vlozegge \\
Floh-Segge \\
Loppe-Star \\
\end{tabular} & $C R$ & $\begin{array}{l}\text { WAT, } \\
\text { AGR, EUT }\end{array}$ & EN & EX & EX & * \\
\hline Carex punctata Gaudin & $\begin{array}{l}\text { Stippeizegge } \\
\text { Punktierte Segge } \\
\text { Prikket Star }\end{array}$ & CR & WAT & SU & $\mathrm{CR}$ & - & - \\
\hline
\end{tabular}




\begin{tabular}{|c|c|c|c|c|c|c|c|}
\hline & & \multirow{2}{*}{$\begin{array}{l}\text { Red List } \\
\text { (trilateral) }\end{array}$} & \multirow[t]{2}{*}{ Threats } & \multicolumn{4}{|c|}{$\begin{array}{l}\text { Status of threat in the subre- } \\
\text { gions of the Wadden Sea Area }\end{array}$} \\
\hline & & & & NL & Nds & SH & DK \\
\hline Carex remota $\mathrm{L}$. & $\begin{array}{l}\text { IJle zegge } \\
\text { Winkel-Segge } \\
\text { Akselblomstret Star }\end{array}$ & su & & SU & - & - & - \\
\hline Carex trinervis Degi. & $\begin{array}{l}\text { Drienervige zegge } \\
\text { Dreinervige Segge } \\
\text { Klit-Star }\end{array}$ & EN & & * & EN & EX & VU \\
\hline Carex vesicaria $\mathrm{L}$. & \begin{tabular}{|l} 
Blaaszegge \\
Blasen-Segge \\
Blære-Star \\
\end{tabular} & Vu & WAT & SU & VU & - & * \\
\hline $\begin{array}{l}\text { Carex viridula }{ }^{\#} \text { Michx. } \\
=\text { C. oederi } \\
=\text { C. serotina Mérat ssp. seroti- } \\
\text { na }\end{array}$ & $\begin{array}{l}\text { Geelgroene zegge I } \\
\text { Dwergzegge } \\
\text { Kleine Gelb-Segge } \\
\text { AErte Star }\end{array}$ & EN & WAT & Vu & VU & CR & - \\
\hline $\begin{array}{l}\text { Carex viridula ssp. oedocar- } \\
p^{\#} \text { (Anderss.) Schmid } \\
=\text { C. demissa Hornem. } \\
=\text { C.tumidicarpa Anderss. } \\
=\text { C.oederi ssp. oedocarpa } \\
\text { Anderss. }\end{array}$ & $\begin{array}{l}\text { Geelgroene zegge } \\
\text { Grüne Segge } \\
\text { Grøn Star }\end{array}$ & * & & SU & - & - & * \\
\hline $\begin{array}{l}\text { Carex viridula var. pulchella }{ }^{\#} \\
\text { (Lönnr.) Schmid } \\
=\text { C.oederi ssp. oederi } \\
=\text { C.oederi ssp. pulchella } \\
=\text { C.scandinavica Davies } \\
=\text { C. serotina Mérat spp. pul- } \\
\text { chella (Lönnr.) } \\
\end{array}$ & $\begin{array}{l}\text { Dwergzegge } \\
\text { Späte Segge } \\
\text { Høst-Star }\end{array}$ & VU & WAT & VU & VU & $\mathrm{CR}$ & * \\
\hline Carlina vulgaris L. s.str. & \begin{tabular}{|l|} 
Driedistel \\
Golddistel \\
Bakketidsel \\
\end{tabular} & EN & HAB,EUT & VU & - & $\mathrm{CR}$ & - \\
\hline Carum carvi L. & \begin{tabular}{|l} 
Echte karwij \\
Wiesen-Kümmel \\
Kommen \\
\end{tabular} & * & & * & VU & * & - \\
\hline $\begin{array}{l}\text { Catabrosa aquatica (L.) } \\
\text { P.Beauv. }\end{array}$ & $\begin{array}{l}\text { Watergras } \\
\text { Quellgras } \\
\text { Tæppegræs } \\
\end{array}$ & CR & WAT & CR & EX & EN & su \\
\hline Centaurium erythraea Rafn & $\begin{array}{l}\text { Echt duizendguldenkruid } \\
\text { Echtes Tausendgülden- } \\
\text { kraut } \\
\text { Mark-Tusindgylden }\end{array}$ & VU & WAT & VU & - & ${ }^{*}$ & SU \\
\hline
\end{tabular}

\# Obviously there is a problem concerning the taxonomy of Carex viridula which could not be solved on the trilateral level. 


\begin{tabular}{|c|c|c|c|c|c|c|c|}
\hline & & \multirow{2}{*}{$\begin{array}{c}\text { Red List } \\
\text { (trilateral) }\end{array}$} & \multirow{2}{*}{ Threats } & \multicolumn{4}{|c|}{$\begin{array}{l}\text { Status of threat in the subre- } \\
\text { gions of the Wadden Sea Area }\end{array}$} \\
\hline & & & & NL & Nds & $\mathrm{SH}$ & DK \\
\hline Cerastium diffusum Pers. & $\begin{array}{l}\text { Scheve hoornbloem } \\
\text { Viermänniges Hornkraut } \\
\text { Firehannet Hønsetarm }\end{array}$ & $\cdot$ & DIS & * & su & su & vu \\
\hline Cerastium pumilum Curtis & $\begin{array}{l}\text { Steenhoornbloem } \\
\text { Dunkles Zwerg-Hornkraut } \\
\text { Liden Hønsetarm }\end{array}$ & SU? & & SU & - & ? & - \\
\hline Chenopodium botryodes Sm. & $\begin{array}{l}\text { Beursjesganzevoet } \\
\text { Dickblättriger Gänsefuß } \\
\text { Drue-Gåsefod }\end{array}$ & EX & ? & EX & - & - & - \\
\hline Cicendia filiformis (L.) Dełarbre & $\begin{array}{l}\text { Draadgentiaan } \\
\text { Heide Zindelkraut } \\
\text { Bitterblad }\end{array}$ & CR & WAT, EUT & EN & EX & - & - \\
\hline Cicuta virosa $\mathrm{L}$. & $\begin{array}{l}\text { Waterscheerling } \\
\text { Wasserschierling } \\
\text { Alm. Gifttyde }\end{array}$ & su? & & SU & - & $(*)$ & - \\
\hline Cirsium dissectum (L.) Hill & $\begin{array}{l}\text { Spaanse ruiter } \\
\text { Englische Kratzdistel } \\
\text { Engelsk Tidsel } \\
\end{array}$ & EN & WAT, AGR & EN & - & - & - \\
\hline Cochlearia anglica $\mathrm{L}$. & $\begin{array}{l}\text { Engelss lepelblad } \\
\text { Englisches Loffelkraut } \\
\text { Engelsk Kokleare } \\
\end{array}$ & * & $\begin{array}{c}\text { AGR, DIS } \\
\text { WAT }\end{array}$ & * & * & VU & - \\
\hline Cochlearia officinalis $\mathrm{L}$. & $\begin{array}{l}\text { Echt lepelblad } \\
\text { Gebräuchliches Löffelkraut } \\
\text { Læge Kokleare }\end{array}$ & CR & WAT & su & $\mathrm{CR}$ & $C R$ & * \\
\hline Cotula coronopifolia L. & $\begin{array}{l}\text { Goudknopje } \\
\text { Krähenfuß-Laugenblume } \\
\text { Firkløft }\end{array}$ & EN & AGR, WAT & su & EN & EN & - \\
\hline Crambe maritima $\mathrm{L}$. & \begin{tabular}{|l} 
Zeekool \\
Meerkohi \\
Strandkăl
\end{tabular} & CR & & su & EX & - & - \\
\hline Crepis tectorum $\mathrm{L}$. & $\begin{array}{l}\text { Smal streepzaad } \\
\text { Dach-Pippau, Mauer-Pip- } \\
\text { pau } \\
\text { Tag-Høgeskæg }\end{array}$ & Vu & AGR & EN & - & * & * \\
\hline Crithmum maritimum $\mathrm{L}$. & $\begin{array}{l}\text { Zeevenkel } \\
\text { Stranddild }\end{array}$ & su & & su & - & - & - \\
\hline Cuscuta epithymum (L.) L. & $\begin{array}{l}\text { Klein warkruid } \\
\text { Quendel-Seide } \\
\text { Lyng-Silke } \\
\end{array}$ & CR & AGR & EX & EN & EN & su \\
\hline Dactylorhiza incarnata (L.) Soo & $\begin{array}{l}\text { Vleeskleurige orchis } \\
\text { Steifblättriges Knabenkraut } \\
\text { Kødfarvet Gøgeurt }\end{array}$ & EN & WAT, EUT & VU & CR & - & * \\
\hline
\end{tabular}




\begin{tabular}{|c|c|c|c|c|c|c|c|}
\hline & & \multirow{2}{*}{$\begin{array}{c}\text { Red List } \\
\text { (trilateral) }\end{array}$} & \multirow[t]{2}{*}{ Threats } & \multicolumn{4}{|c|}{$\begin{array}{l}\text { Status of threat in the subre- } \\
\text { gions of the Wadden Sea Area }\end{array}$} \\
\hline & & & & NL & Nas & $\mathrm{SH}$ & DK \\
\hline Dactylorhiza maculata (L) Soo & $\begin{array}{l}\text { Gevlekte orchis } \\
\text { Geflecktes Knabenkraut } \\
\text { Plettet Gøgeurt }\end{array}$ & Vu & HAB, AGR & Vu & Vu & VU & * \\
\hline $\begin{array}{l}\text { Dactylorhiza majalis (Rchb.) } \\
\text { Hunt \& Summerh. }\end{array}$ & \begin{tabular}{|l|} 
Breedbladige orchis \\
Breitblăttriges Knabenkraut \\
Maj-Gøgeurt
\end{tabular} & Vu & $\begin{array}{c}\text { EUT, WAT, } \\
\text { AGR }\end{array}$ & Vu & EN & EN & * \\
\hline $\begin{array}{l}\text { Dactylorhiza praetermissa } \\
\text { (Druce) Soo }\end{array}$ & \begin{tabular}{|l|} 
Rietorchis \\
Übersehenes Knabenkraut \\
Priklæbet Gøgeurt
\end{tabular} & Vu & $\begin{array}{c}\text { EUT, WAT, } \\
\text { AGR }\end{array}$ & vu & su & - & - \\
\hline $\begin{array}{l}\text { Deschampsia setacea (Huds.) } \\
\text { Hackel }\end{array}$ & $\begin{array}{l}\text { Moerassmele } \\
\text { Borst-Schmiele } \\
\text { Fin Bunke } \\
\end{array}$ & CR & WAT, EUT & CR & - & CR & su \\
\hline $\begin{array}{l}\text { Deschampsia wibeliana (Son- } \\
\text { der) Part. }\end{array}$ & Schlamm-Schmiele & su & HAB, WAT & - & su & su & - \\
\hline Desmazeria marina (L.) Druce & Laksteeltje & su & & su & - & - & - \\
\hline Dianthus carthusianorum L. & \begin{tabular}{|l} 
Karthuizer anjer \\
Karthäuser-Nelke \\
Karteuser-Nellike
\end{tabular} & CR & HAB, EUT & - & - & $\mathrm{CR}$ & - \\
\hline Dianthus deltoides L. & $\begin{array}{l}\text { Steenanjer } \\
\text { Heide-Nelke } \\
\text { Bakke-Nellike }\end{array}$ & CR & HAB, EUT & SU & - & $\mathrm{CR}$ & - \\
\hline Diplotaxis muralis (L.) DC. ${ }^{1}$ & $\begin{array}{l}\text { Kleine zandkool } \\
\text { Mauer-Doppelsame } \\
\text { Mursennep }\end{array}$ & * & & su & * & $\left({ }^{*}\right)$ & $(*)$ \\
\hline Drosera intermedia Hayne & $\begin{array}{l}\text { Kleine zonnedauw } \\
\text { Mittlerer Sonnentau } \\
\text { Liden Soldug }\end{array}$ & EN & WAT & $\mathrm{CR}$ & - & Vu & * \\
\hline Drosera rotundifolia L. & $\begin{array}{l}\text { Ronde zonnedauw } \\
\text { Rundblättriger Sonnentau } \\
\text { Rundbladet Soldug }\end{array}$ & * & WAT, AGR & * & VU & * & * \\
\hline $\begin{array}{l}\text { Eleocharis acicularis (L.) Roem. } \\
\text { \& Schult. }\end{array}$ & \begin{tabular}{|l} 
Naaldwaterbies \\
Nadel-Sumpfsimse \\
Nåle-Sumpstrå
\end{tabular} & CR & WAT, EUT & su & EX & EN & su \\
\hline $\begin{array}{l}\text { Eleocharis multicaulis (Sm.) } \\
\mathrm{Sm} \text {. }\end{array}$ & \begin{tabular}{|l|} 
Veelstengelige waterbies \\
Vielstengelige Sumpfsimse \\
Mangestænglet Sumpstrå
\end{tabular} & Vu & $\begin{array}{l}\text { WAT, EUT, } \\
\text { AGR, HAB }\end{array}$ & * & - & $\mathrm{CR}$ & * \\
\hline $\begin{array}{l}\text { Eleocharis quinqueflora } \\
\text { (F.X.Hartmann) O. Schwarz }\end{array}$ & $\begin{array}{l}\text { Armbloemige waterbies } \\
\text { Wenigblütige Sumpfbinse } \\
\text { Fåblomstret Kogleaks }\end{array}$ & Vu & WAT & vu & EN & EN & * \\
\hline
\end{tabular}




\begin{tabular}{|c|c|c|c|c|c|c|c|}
\hline & & \multirow{2}{*}{$\begin{array}{l}\text { Red List } \\
\text { (trilateral) }\end{array}$} & \multirow[t]{2}{*}{ Threats } & \multicolumn{4}{|c|}{$\begin{array}{l}\text { Status of threat in the subre- } \\
\text { gions of the Wadden Sea Area }\end{array}$} \\
\hline & & & & NL & Nds & $\mathrm{SH}$ & DK \\
\hline $\begin{array}{l}\text { Epipactis palustris (L.) } \\
\text { Crantz. }\end{array}$ & $\begin{array}{l}\text { Moeraswespenorchis } \\
\text { Echter Sumpfwurz } \\
\text { Sump-Hullæbe }\end{array}$ & $\dot{E N}$ & WAT & VU & EN & $C R$ & * \\
\hline $\begin{array}{l}\text { Equisetum variegatum } \\
\text { Schleicher ex. Web. \& Mohr }\end{array}$ & $\begin{array}{l}\text { Bonte paardestaart } \\
\text { Bunter Schachtelhalm } \\
\text { Liden Padderokke }\end{array}$ & CR & WAT & CR & CR & - & - \\
\hline Erica cinerea L. & $\begin{array}{l}\text { Rode dophei } \\
\text { Grau-Heide } \\
\text { Grälyng } \\
\end{array}$ & EX & $?$ & EX & - & - & - \\
\hline Erica scoparia L. & Bezemdopheide & EN & $?$ & EN & - & - & - \\
\hline Eriophorum vaginatum $\mathrm{L}$. & $\begin{array}{l}\text { Eenarig wollegras } \\
\text { Scheidiges Wollgras } \\
\text { Tue-Kæruld }\end{array}$ & CR & WAT & EX & EX & - & * \\
\hline Eryngium maritimum $\mathrm{L}$. & $\begin{array}{l}\text { Blauwe zeedistel } \\
\text { Stranddistel } \\
\text { Strand-Mandstro }\end{array}$ & Vu & DIS, WAT & VU & EN & VU & - \\
\hline Euphorbia paralias L. & $\begin{array}{l}\text { Zeewolfsmelk } \\
\text { Kilt-Vertemælk }\end{array}$ & CR & DIS & CR & - & - & - \\
\hline Euphrasia micrantha Rchb. & $\begin{array}{l}\text { Slanke ogentroost } \\
\text { Schlanker Augentrost } \\
\text { Lyng-Øjentrøst }\end{array}$ & EN & $\begin{array}{l}\text { AGR, HAB, } \\
\text { EUT, AFF }\end{array}$ & - & EN & CR & * \\
\hline $\begin{array}{l}\text { Euphrasia stricta D. Woiff ex. } \\
\text { J.F.Lehm. }\end{array}$ & $\begin{array}{l}\text { Stijve ogentroost } \\
\text { Steifer Augentrost } \\
\text { Spids Øjentrøst }\end{array}$ & Vu & $\begin{array}{c}\text { AGR, HAB, } \\
\text { EUT }\end{array}$ & * & Vu & vu & * \\
\hline Filago lutescens Jord. ${ }^{1}$ & $\begin{array}{l}\text { Geel viltkruid } \\
\text { Gelbliches Filzkraut } \\
\text { Gulgrå Museurt }\end{array}$ & EX? & & - & - & EX & $\left({ }^{*}\right)$ \\
\hline Filago minima (Sm.) Pers. & \begin{tabular}{|l} 
Dwergviltkruid \\
Zwerg-Filzkraut \\
Liden Museurt \\
\end{tabular} & VU & $\begin{array}{l}\text { HAB, EUT, } \\
\text { AFF }\end{array}$ & EN & Vu & * & * \\
\hline Filago vulgaris Lam. & $\begin{array}{l}\text { Duits viltkruid } \\
\text { Deutsches Filzkraut } \\
\text { Kugle-Museurt } \\
\end{array}$ & CR & $\begin{array}{l}\text { HAB, EUT, } \\
\text { AFF }\end{array}$ & CR & - & CR & * \\
\hline Galeopsis segetum Necker & $\begin{array}{l}\text { Bleekgele hennepnetel } \\
\text { Saat-Hohlzahn } \\
\text { Gul Hanekro } \\
\end{array}$ & su & & su & - & - & - \\
\hline Galeopsis speciosa Mill. & $\begin{array}{l}\text { Dauwnetel } \\
\text { Bunter Hohizahn } \\
\text { Hamp-Hanekro }\end{array}$ & VU? & & SU & vu & $(*)$ & - \\
\hline
\end{tabular}




\begin{tabular}{|c|c|c|c|c|c|c|c|}
\hline & & \multirow{2}{*}{$\begin{array}{c}\text { Red List } \\
\text { (trilateral) }\end{array}$} & \multirow[t]{2}{*}{ Threats } & \multicolumn{4}{|c|}{$\begin{array}{l}\text { Status of threat in the subre- } \\
\text { gions of the Wadden Sea Area }\end{array}$} \\
\hline & & & & NL & Nds & $\mathrm{SH}$ & DK \\
\hline Galium sterneri Ehrendf. & $\begin{array}{l}\text { Sterners Labkraut } \\
\text { Liden Snerre } \\
\end{array}$ & EN & EUT & - & - & $\mathrm{CR}$ & * \\
\hline Genista germanica L. & $\begin{array}{l}\text { Duitse brem } \\
\text { Deutscher Ginster } \\
\text { Tysk Visse }\end{array}$ & EX & & - & - & EX & EX \\
\hline Genista tinctoria L. & $\begin{array}{l}\text { Verfbrem } \\
\text { Färber-Ginster } \\
\text { Farve-Visse }\end{array}$ & * & AGR, EUT & Vu & - & * & * \\
\hline Gentiana pneumonanthe $\mathrm{L}$. & $\begin{array}{l}\text { Klokjesgentiaan } \\
\text { Lungen-Enzian } \\
\text { Klokke-Ensian }\end{array}$ & EN & WAT, AGR & VU & EX & $C R$ & * \\
\hline $\begin{array}{l}\text { Gentianella campestris } \\
\text { ssp.baltica (Murb.) A. \& D. Love }\end{array}$ & $\begin{array}{l}\text { Veldgentiaan (ssp. baltica) } \\
\text { Baltischer Enzian } \\
\text { Baltisk Ensian }\end{array}$ & CR & WAT & CR & $\mathrm{CR}$ & EX & VU \\
\hline $\begin{array}{l}\text { Gentianella uliginosa (Willd) } \\
\text { Borner }\end{array}$ & $\begin{array}{l}\text { Slanke gentiaan } \\
\text { Sumpf-Enzian } \\
\text { Eng-Ensian } \\
\end{array}$ & EN & WAT & EN & $C R$ & - & VU \\
\hline Gnaphalium luteo-album L. & $\begin{array}{l}\text { Bleekgele droogbloem } \\
\text { Gelbweißes Ruhrkraut } \\
\text { Gulhvid Evighedsblomst }\end{array}$ & CR & WAT & $C R$ & EX & - & - \\
\hline $\begin{array}{l}\text { Gymnadenia conopsea (L.) } \\
\text { R.Br. }\end{array}$ & $\begin{array}{l}\text { Grote muggenorchis } \\
\text { Große-Händelwurz } \\
\text { Langakset Trådspore }\end{array}$ & EN & WAT & EN & CR & - & - \\
\hline $\begin{array}{l}\text { Halimione pedunculata (L.) } \\
\text { Aellen }\end{array}$ & $\begin{array}{l}\text { Gesteelde zoutmelde } \\
\text { Gestielte Keilmelde } \\
\text { Stilket Kilebæger } \\
\end{array}$ & EN & DIS, AGR & * & EN & $\mathrm{CR}$ & * \\
\hline $\begin{array}{l}\text { Halimione portulacoides (L.) } \\
\text { Aellen } \\
\text { (= Atriplex portulacoides) }\end{array}$ & $\begin{array}{l}\text { Gewone zoutmelde } \\
\text { Strand-Salzmelde } \\
\text { Stilkløs Kilebæger }\end{array}$ & VU & $\begin{array}{c}\text { DIS, AGR } \\
\text { HAB }\end{array}$ & * & * & EN & * \\
\hline $\begin{array}{l}\text { Hammarbya paludosa } \\
\text { (L.) O. Kuntze }\end{array}$ & $\begin{array}{l}\text { Veenmosorchis } \\
\text { Sumpf-Weichwurz } \\
\text { Femkantet Hjertelæbe }\end{array}$ & CR & WAT, EUT & EX & EX & $C R$ & SU \\
\hline $\begin{array}{l}\text { Helictotrichon pubescens } \\
\text { (Huds.) Pilger }\end{array}$ & $\begin{array}{l}\text { Zachte haver } \\
\text { Flaumiger Wiesenhafer } \\
\text { Dunet Havre }\end{array}$ & EN & AGR, EUT & su & - & EN & - \\
\hline $\begin{array}{l}\text { Herminium monorchis (L.) } \\
\text { R.Br. }\end{array}$ & $\begin{array}{l}\text { Honingorchis } \\
\text { Einknollige Honigorchis, } \\
\text { Einknolle } \\
\text { Pukkellæbe }\end{array}$ & CR & WAT & $\mathrm{CR}$ & - & - & - \\
\hline Herniaria glabra L. & $\begin{array}{l}\text { Breukkruid } \\
\text { Bruchkraut } \\
\text { Brudurt }\end{array}$ & * & & - & - & su & * \\
\hline
\end{tabular}




\begin{tabular}{|c|c|c|c|c|c|c|c|}
\hline & & \multirow{2}{*}{$\begin{array}{c}\text { Red List } \\
\text { (trilateral) }\end{array}$} & \multirow[t]{2}{*}{ Threats } & \multicolumn{4}{|c|}{$\begin{array}{l}\text { Status of threat in the subre- } \\
\text { gions of the Wadden Sea Area }\end{array}$} \\
\hline & & & & NL & Nds & $\mathrm{SH}$ & DK \\
\hline Hieracium vulgatum Fries & $\begin{array}{l}\text { Dicht havikskruid } \\
\text { Gemeines Habichtskraut } \\
\text { Almindelig Høgeurt }\end{array}$ & EX & $?$ & EX & - & - & - \\
\hline $\begin{array}{l}\text { Hierochloe odorata (L.) P.Be- } \\
\text { auv. }\end{array}$ & \begin{tabular}{|l} 
Veenreukgras \\
Duft-Mariengras \\
Vellugtende Festgræs
\end{tabular} & vu & & * & EN & - & * \\
\hline Hordeum marinum Huds. & \begin{tabular}{|l} 
Zeegerst \\
Strand-Gerste \\
Strand-Byg \\
\end{tabular} & CR & WAT, AGR & EN & EX & EX & - \\
\hline Hordeum secalinum Schreb. & $\begin{array}{l}\text { Veldgerst } \\
\text { Roggen-Gerste } \\
\text { Eng-Byg }\end{array}$ & * & & * & VU & * & * \\
\hline Hottonia palustris L. & $\begin{array}{l}\text { Waterviolier } \\
\text { Wasserfeder } \\
\text { Vandrøllike }\end{array}$ & CR & WAT & EX & - & - & * \\
\hline $\begin{array}{l}\text { Huperzia selago (L.) } \\
\text { Schrank \& Mart. }\end{array}$ & $\begin{array}{l}\text { Dennewolfsklauw } \\
\text { Otteradet Ulvefod }\end{array}$ & CR & EUT & su & $\mathrm{CR}$ & - & - \\
\hline Hypericum humifusum L. & $\begin{array}{l}\text { Liggend hertshooi } \\
\text { Liegendes Hartheu } \\
\text { Dværg-Perikon }\end{array}$ & EX? & WAT, AGR & EX & EX & $\left({ }^{*}\right)$ & EX? \\
\hline Hypericum maculatum Crantz & $\begin{array}{l}\text { Kantig hertshooi } \\
\text { Kanten-Hartheu } \\
\text { Kantet Perikon }\end{array}$ & • & & su & * & su & * \\
\hline Hypericum pulchrum L. & $\begin{array}{l}\text { Fraai hertshooi } \\
\text { Schönes Johanniskraut } \\
\text { Smuk Perikon }\end{array}$ & EN & & su & vu & CR & - \\
\hline Hypochoeris glabra L. & $\begin{array}{l}\text { Glad biggekruid } \\
\text { Kahles Ferkelkraut } \\
\text { Glat Kongepen }\end{array}$ & EN & $\begin{array}{c}\text { HAB, EUT, } \\
\text { AFF }\end{array}$ & $\mathrm{CR}$ & EN & Vu & su \\
\hline Illecebrum verticillatum $\mathrm{L}$. & $\begin{array}{l}\text { Grondster } \\
\text { Quirlige Knorpelmiere } \\
\text { Bruskbæger }\end{array}$ & EX & WAT & EX & - & - & - \\
\hline Inula britannica L. & $\begin{array}{l}\text { Engelse alant } \\
\text { Wiesen-Alant } \\
\text { Soløje-Alant } \\
\end{array}$ & VU & WAT & VU & VU & - & " \\
\hline Isolepis fluitans (L.) R.Br. & $\begin{array}{l}\text { Vlotende bies } \\
\text { Flutende Schuppensimse } \\
\text { Flydende Kogleaks }\end{array}$ & EN & $\begin{array}{c}\text { WAT, } \\
\text { AGR, EUT, } \\
\text { HAB } \\
\end{array}$ & Vu & - & $C R$ & su \\
\hline Juncus anceps Laharpe & $\begin{array}{l}\text { Duinrus } \\
\text { Zweischneidige Binse } \\
\text { Sand-Siv }\end{array}$ & vu & WAT & * & * & EN & * \\
\hline
\end{tabular}




\begin{tabular}{|c|c|c|c|c|c|c|c|}
\hline & & \multirow{2}{*}{$\begin{array}{c}\text { Red List } \\
\text { (trilateral) }\end{array}$} & \multirow[t]{2}{*}{ Threats } & \multicolumn{4}{|c|}{$\begin{array}{l}\text { Status of threat in the subre- } \\
\text { gions of the Wadden Sea Area }\end{array}$} \\
\hline & & & & $\mathrm{NL}$ & Nds & $\mathrm{SH}$ & DK \\
\hline Juncus balticus willd. & $\begin{array}{l}\text { Noordse rus } \\
\text { Baltische Binse } \\
\text { Klit-Siv }\end{array}$ & vu & & * & $\mathrm{CR}$ & - & * \\
\hline $\begin{array}{l}\text { Juncus bulbosus ssp. kochii } \\
\text { (F.W.Schultz) Reichg. }\end{array}$ & $\begin{array}{l}\text { Kleine knolrus } \\
\text { Zwiebel-Binse } \\
\text { Opret Siv }\end{array}$ & SU? & & su & - & - & $?$ \\
\hline Juncus capitatus Weigel & $\begin{array}{l}\text { Koprus } \\
\text { Kopf-Binse } \\
\text { Fin Siv } \\
\end{array}$ & CR & $\begin{array}{l}\text { WAT, } \\
\text { AGR, EUT }\end{array}$ & EX & EX & $\mathrm{CR}$ & VU \\
\hline Juncus inflexus L. & \begin{tabular}{|l} 
Zeegroene rus \\
Blaugrüne Binse \\
Blågrå Siv
\end{tabular} & vu & WAT & Vu & * & - & - \\
\hline Juncus maritimus Lam. & $\begin{array}{l}\text { Zeerus } \\
\text { Strand-Binse } \\
\text { Strand-Siv }\end{array}$ & * & AGR & * & * & su & * \\
\hline Juncus pygmaeus L. & $\begin{array}{l}\text { Dwergrus } \\
\text { Zwerg-Binse } \\
\text { Dværg-Siv }\end{array}$ & EN & $\begin{array}{l}\text { WAT, } \\
\text { AGR, EUT }\end{array}$ & EN & - & $\mathrm{CR}$ & * \\
\hline Juniperus communis $\mathrm{L}$. & $\begin{array}{l}\text { Jeneverbes } \\
\text { Gemeiner Wacholder } \\
\text { Alm. Ene }\end{array}$ & CR & AGR & $\mathrm{CR}$ & - & $\mathrm{CR}$ & * \\
\hline Koeleria arenaria (Dum.) Conert & $\begin{array}{l}\text { Smal fakkelgras } \\
\text { Sand-Schillergras } \\
\text { Klit-Kambunke }\end{array}$ & * & & * & VU & - & * \\
\hline $\begin{array}{l}\text { Lactuca tatarica (L.) } \\
\text { C.A.Meyer }\end{array}$ & $\begin{array}{l}\text { Strandsla } \\
\text { Tataren-Lattich } \\
\text { Strand-Salat } \\
\end{array}$ & SU & & su & - & - & - \\
\hline $\begin{array}{l}\text { Lathyrus linifolius (Reichard) } \\
\text { Bässler }\end{array}$ & $\begin{array}{l}\text { Knollathyrus } \\
\text { Berg-Platterbse } \\
\text { Krat-Fladbælg }\end{array}$ & EN & & - & - & EN & - \\
\hline $\begin{array}{l}\text { Lathyrus maritimus (L.) } \\
\text { Bigel. (=L. japonicus Willd.) }\end{array}$ & $\begin{array}{l}\text { Zeelathyrus } \\
\text { Strand-Platterbse } \\
\text { Strandært } \\
\end{array}$ & vu & WAT, DIS & su & EN & * & * \\
\hline Lepidium campestre (L.) R.Br.. ${ }^{1}$ & $\begin{array}{l}\text { Veldkruidkers } \\
\text { Feld-Kresse } \\
\text { Salomons Lysestage }\end{array}$ & vu & & su & VU & * & $(*)$ \\
\hline Limonium vulgare Mill. & $\begin{array}{l}\text { Lamsoor } \\
\text { Gewöhnlicher Strandflieder } \\
\text { Tætblomstret Hindebæger }\end{array}$ & * & & * & Vu & * & * \\
\hline Limosella aquatica $L$. & $\begin{array}{l}\text { Slijkgroen } \\
\text { Schlammkraut } \\
\text { Dyndurt }\end{array}$ & EN & WAT & $\mathrm{CR}$ & Vu & $C R$ & - \\
\hline
\end{tabular}




\begin{tabular}{|c|c|c|c|c|c|c|c|}
\hline & & \multirow{2}{*}{$\begin{array}{l}\text { Red List } \\
\text { (trilateral) }\end{array}$} & \multirow[t]{2}{*}{ Threats } & \multicolumn{4}{|c|}{$\begin{array}{l}\text { Status of threat in the subre- } \\
\text { gions of the Wadden Sea Area }\end{array}$} \\
\hline & & & & NL & Nds & $\mathrm{SH}$ & DK \\
\hline Linum catharticum L. & $\begin{array}{l}\text { Geelhartje } \\
\text { Purgier-Lein } \\
\text { Vild Hør }\end{array}$ & ivu & AGR, WAT & $\star$ & VU & Vu & * \\
\hline Liparis loeselii (L.) L.C.M.Richard & $\begin{array}{l}\text { Groenknolorchis } \\
\text { Sumpf-Glanzkraut } \\
\text { Mygblomst }\end{array}$ & EN & WAT & EN & $\mathrm{CR}$ & - & - \\
\hline Listera cordata (L.) R.Br. & $\begin{array}{l}\text { Kleoine keverorchis } \\
\text { Kleines Zweiblatt } \\
\text { Hjertebladet Fliglæbe }\end{array}$ & EN & & * & - & EX & - \\
\hline Lithospermum officinale $\mathrm{L}$. & $\begin{array}{l}\text { Glad parelzaad } \\
\text { Echter Steinsame } \\
\text { Læge-Stenfrø } \\
\end{array}$ & su & & su & - & - & - \\
\hline Littorella uniflora (L.) Asch. & $\begin{array}{l}\text { Oeverkruid } \\
\text { See-Strandling } \\
\text { Strandbo }\end{array}$ & EN & WAT, EUT & EN & EN & $\mathrm{CR}$ & - \\
\hline Lotus glaber Mill. & $\begin{array}{l}\text { Smalle rolklaver } \\
\text { Salz-Hornklee } \\
\text { Smalbladet Kællingetand }\end{array}$ & * & & * & Vu & - & * \\
\hline $\begin{array}{l}\text { Lycopodiella inundata } \\
\text { (L.) Holub }\end{array}$ & $\begin{array}{l}\text { Moeraswolfsklauw } \\
\text { Gemeiner Moorbärlapp } \\
\text { Liden Ulvefod }\end{array}$ & Vu & WAT & * & VU & EN & * \\
\hline Lycopodium clavatum L. & $\begin{array}{l}\text { Grote woifsklauw } \\
\text { Keulen-Bärlapp } \\
\text { Alm. Ulvefod }\end{array}$ & VU & $\begin{array}{l}\text { AGR, HAB, } \\
\text { EUT, AFF }\end{array}$ & SU & Vu & EN & su \\
\hline Lysimachia thyrsiflora $\mathrm{L}$. & $\begin{array}{l}\text { Moeraswederik } \\
\text { Strauß-Gilbweiderich } \\
\text { Dusk-Fredløs } \\
\end{array}$ & EN? & WAT & EX & Vu & $(*)$ & * \\
\hline Marrubium vulgare $L_{. .}{ }^{1}$ & \begin{tabular}{|l} 
Malrove \\
Gemeiner Andorn \\
Kransburre
\end{tabular} & EX? & AGR & EX & - & - & $?$ \\
\hline Menyanthes trifoliata $\mathrm{L}$. & $\begin{array}{l}\text { Waterdrieblad } \\
\text { Dreiblattriger Fieberklee } \\
\text { Bukkeblad }\end{array}$ & vu & WAT & VU & EN & vu & * \\
\hline Myosurus minimus L... & $\begin{array}{l}\text { Muizestaart } \\
\text { Mäuseschwänzchen } \\
\text { Musehale }\end{array}$ & EN? & AGR & $\mathrm{CR}$ & Vu & $\left({ }^{*}\right)$ & $(*)$ \\
\hline $\begin{array}{l}\text { Myriophyllum alterniflorum } \\
\text { DC. }\end{array}$ & $\begin{array}{l}\text { Teer vederkruid } \\
\text { Wechselblütiges Tausend- } \\
\text { blatt } \\
\text { Hår-Tusindblad } \\
\end{array}$ & EN & WAT, EUT & VU & $\mathrm{EX}$ & - & * \\
\hline $\begin{array}{l}\text { Narthecium ossifragum (L.) } \\
\text { Huds. }\end{array}$ & \begin{tabular}{|l} 
Beenbreek \\
Beinbrech \\
Benbræk
\end{tabular} & EN & WAT & - & - & $\mathrm{CR}$ & * \\
\hline
\end{tabular}




\begin{tabular}{|c|c|c|c|c|c|c|c|}
\hline & & \multirow{2}{*}{$\begin{array}{c}\text { Red List } \\
\text { (trilateral) }\end{array}$} & \multirow[t]{2}{*}{ Threats } & \multicolumn{4}{|c|}{$\begin{array}{l}\text { Status of threat in the subre- } \\
\text { gions of the Wadden Sea Area }\end{array}$} \\
\hline & & & & NL & Nds & $\mathrm{SH}$ & DK \\
\hline Odontites litoralis (Fries) Fries & $\begin{array}{l}\text { Vroege ogentroost } \\
\text { Salz-Zahntrost } \\
\text { Strand-Røttop }\end{array}$ & EN & WAT & * & CR & * & - \\
\hline $\begin{array}{l}\text { Oenanthe lachenalii } \\
\text { C.C.Gmelin. }\end{array}$ & $\begin{array}{l}\text { Zilt torkruid } \\
\text { Wiesen-Pferdesaat } \\
\text { Eng-Klaseskærm }\end{array}$ & EN & & * & EN & CR & - \\
\hline $\begin{array}{l}\text { Oenothera ammophila Focke } \\
=0 . \text { oakesiana (A.Gray) } \\
\text { Robbins ex. S. Watson \& Coult }\end{array}$ & $\begin{array}{l}\text { Zandteunisbloem } \\
\text { Dünen-Nachtkerze } \\
\text { Klit-Natlys }\end{array}$ & * & & * & * & * & VU \\
\hline Ophioglossum vulgatum L. & $\begin{array}{l}\text { Addertong } \\
\text { Gewöhnliche Natternzunge } \\
\text { Alm. Slangetunge }\end{array}$ & vu & AGR, WAT & * & EN & EN & * \\
\hline Orchis morio L. & $\begin{array}{l}\text { Harlekijn } \\
\text { Kleines Knabenkraut/ Sa- } \\
\text { lep Knabenkraut } \\
\text { Salep-Gøgeurt } \\
\end{array}$ & CR & WAT, AGR & Vu & EX & - & - \\
\hline Osmunda regalis $\mathrm{L}$. & $\begin{array}{l}\text { Koningsvaren } \\
\text { Königsfarn } \\
\text { Kongebregne } \\
\end{array}$ & Vu & WAT & * & Vu & VU & su \\
\hline $\begin{array}{l}\text { Parapholis strigosa (Dumort.) } \\
\text { Hubb. }\end{array}$ & $\begin{array}{l}\text { Dunstaart } \\
\text { Gekrümmter Dünnschwanz } \\
\text { Spidshale }\end{array}$ & Vu & AGR & * & VU & Vu & * \\
\hline Parnassia palustris L. & $\begin{array}{l}\text { Parnassia } \\
\text { Sumpf-Herzblatt } \\
\text { Alm. Leverurt }\end{array}$ & vu & WAT & Vu & $C R$ & - & * \\
\hline Pedicularis palustris $\mathrm{L}$. & $\begin{array}{l}\text { Moeraskartelblad } \\
\text { Sumpf-Läusekraut } \\
\text { Eng-Troldurt }\end{array}$ & EN & WAT & Vu & $C R$ & $C R$ & * \\
\hline Pedicularis sylvatica L. & $\begin{array}{l}\text { Heidekartelblad } \\
\text { Wald-Läusekraut } \\
\text { Mose-Troldurt } \\
\end{array}$ & EN & $\begin{array}{l}\text { AGR, EUT, } \\
\text { HAB, WAT }\end{array}$ & VU & EX & Vu & * \\
\hline Peplis portula L. & $\begin{array}{l}\text { Waterpostelein } \\
\text { Sumpfquendel } \\
\text { Vandportulak }\end{array}$ & VU & WAT, HAB & * & $\mathrm{VU}$ & EN & su \\
\hline $\begin{array}{l}\text { Peucedanum palustre } \\
\text { (L.) Moench }\end{array}$ & $\begin{array}{l}\text { Melkeppe } \\
\text { Sumpf-Haarstrang, Olse- } \\
\text { nich } \\
\text { Kær-Svovirod }\end{array}$ & * & & su & * & - & * \\
\hline Phleum arenarium L. & $\begin{array}{l}\text { Zanddoddegras } \\
\text { Sand-Lieschgras } \\
\text { Sand-Rottehale }\end{array}$ & * & & * & Vu & - & * \\
\hline Pilularia globulifera L. & \begin{tabular}{|l} 
Pilvaren \\
Pillenfarn \\
Pilledrager
\end{tabular} & CR & $\begin{array}{l}\text { HAB, WAT, } \\
\text { AGR, EUT }\end{array}$ & CR & EX & CR & Vu \\
\hline
\end{tabular}




\begin{tabular}{|c|c|c|c|c|c|c|c|}
\hline & & \multirow{2}{*}{$\begin{array}{c}\text { Red List } \\
\text { (trilateral) }\end{array}$} & \multirow[t]{2}{*}{ Threats } & \multicolumn{4}{|c|}{$\begin{array}{l}\text { Status of threat in the subre- } \\
\text { gions of the Wadden Sea Area }\end{array}$} \\
\hline & & & & NL & Nds & $\mathrm{SH}$ & DK \\
\hline Pimpinella saxifraga $\mathrm{L}$. & $\begin{array}{l}\text { Kleine bevernel } \\
\text { Kleine Pimpinelle } \\
\text { Alm. Pimpinelle } \\
\end{array}$ & - & & su & * & * & * \\
\hline Pinguicula vulgaris $\mathrm{L}$. & $\begin{array}{l}\text { Vetblad } \\
\text { Echtes Fettkraut } \\
\text { Alm. Vibefedt }\end{array}$ & CR & & - & CR & EX & SU \\
\hline $\begin{array}{l}\text { Platanthera bifolia (L.) } \\
\text { L.C.M.Richard }\end{array}$ & $\begin{array}{l}\text { Welriekende nachtorchis } \\
\text { Zweiblättrige Waldhyazint- } \\
\text { he } \\
\text { Bakke-Gøgelilje }\end{array}$ & EN & $\begin{array}{c}E U T, A G R \\
H A B\end{array} \mid$ & VU & EN & CR & * \\
\hline Poa palustris L.. ${ }^{3}$ & $\begin{array}{l}\text { Moerasbeemdgras } \\
\text { Sumpf-Rispe } \\
\text { Stortoppet Ræpgræs }\end{array}$ & * & & su & * & $(*)$ & * \\
\hline Polygala serpyllifolia Hose & $\begin{array}{l}\text { Liggende vleugeltjesbloem } \\
\text { Quendel-Kreuzblümchen } \\
\text { Spæd Mæikeurt }\end{array}$ & EN & & - & - & EX & * \\
\hline Polygala vulgaris $\mathrm{L}$. & $\begin{array}{l}\text { Gewone vleugeltjesbloem } \\
\text { Gewöhnliche Kreuzblume } \\
\text { Alm. Mælkeurt }\end{array}$ & Vu & EUT: & VU & VU & * & * \\
\hline $\begin{array}{l}\text { Polygonatum odoratum (Mill) } \\
\text { Druce }\end{array}$ & $\begin{array}{l}\text { Welriekende salomonsze- } \\
\text { gel } \\
\text { Duftende Weißwurz, Sa- } \\
\text { lomonssiegel } \\
\text { Kantet Konval }\end{array}$ & SU & & SU & - & - & - \\
\hline Polygonum minus Huds. & \begin{tabular}{|l} 
Kleine duizendknoop \\
Kleiner Knöterich \\
Liden Pileurt \\
\end{tabular} & * & & su & * & $(*)$ & * \\
\hline $\begin{array}{l}\text { Polygonum oxyspermum Mey- } \\
\text { er \& Bunge ssp. raii (Bab.) } \\
\text { Webb \& Chater }\end{array}$ & $\begin{array}{l}\text { Zandduizenknoop } \\
\text { Sand-Knöterich } \\
\text { Sand-Pileurt }\end{array}$ & EN & & - & - & $\mathrm{CR}$ & Vu \\
\hline $\begin{array}{l}\text { Potamogeton coloratus } \\
\text { Hornem. }\end{array}$ & $\begin{array}{l}\text { Weegbreefonteinkruid } \\
\text { Gefärbtes Laichkraut } \\
\text { Vejbred-Vandaks }\end{array}$ & su & & SU & - & - & - \\
\hline Potamogeton gramineus $\mathrm{L}$. & $\begin{array}{l}\text { Ongelijkbladig fonteinkruid } \\
\text { Gras-Laichkraut } \\
\text { Græsbladet Vandaks }\end{array}$ & EN & WAT, EUT & EN & $E N$ & su & * \\
\hline Potamogeton perfoliatus L. & $\begin{array}{l}\text { Doorgroeid fonteinkruid } \\
\text { Durchwachsenes Laich- } \\
\text { kraut } \\
\text { Hjertebladet Vandaks }\end{array}$ & EN & WAT & EX & - & su & * \\
\hline $\begin{array}{l}\text { Potamogeton polygonifolius } \\
\text { Pourr. }\end{array}$ & \begin{tabular}{|l} 
Duizendknoopfonteinkruid \\
Knöterich-Laichkraut \\
Aflangbladet Vandaks \\
\end{tabular} & Vu & $\begin{array}{c}\text { WAT, EUT, } \\
\text { AGR }\end{array}$ & * & Vu & VU & * \\
\hline
\end{tabular}




\begin{tabular}{|c|c|c|c|c|c|c|c|}
\hline & & \multirow{2}{*}{$\begin{array}{c}\text { Red List } \\
\text { (trilateral) }\end{array}$} & \multirow[t]{2}{*}{ Threats } & \multicolumn{4}{|c|}{$\begin{array}{l}\text { Status of threat in the subre- } \\
\text { gions of the Wadden Sea Area }\end{array}$} \\
\hline & & & & $\mathrm{NL}$ & Nds & $\mathrm{SH}$ & DK \\
\hline $\begin{array}{l}\text { Potamogeton trichoides } \\
\text { Cham. \& Schlechtend. }\end{array}$ & $\begin{array}{l}\text { Haarfonteinkruid } \\
\text { Haarblättriges Laichkraut } \\
\text { Hårfin Vandaks }\end{array}$ & Vu & WAT & VU & - & - & * \\
\hline Potentilla argentea $\mathrm{L}$. & $\begin{array}{l}\text { Viltganzerik } \\
\text { Silber-Fingerkraut } \\
\text { Solv-Potentił }\end{array}$ & vu & ? & EX & * & $*$ & $*$ \\
\hline $\begin{array}{l}\text { Puccinellia capillaris } \\
\text { (Liljeblad) Jansen }\end{array}$ & $\begin{array}{l}\text { Bleek kweldergras } \\
\text { Haar-Salzschwaden } \\
\text { Slap Annelgræs }\end{array}$ & * & & SU & SU & * & * \\
\hline Pyrola minor L. & $\begin{array}{l}\text { Klein wintergroen } \\
\text { Kleines Wintergrün } \\
\text { Liden Vintergrøn }\end{array}$ & vu & ? & VU & VU & VU & - \\
\hline Pyrola rotundifolia L. & $\begin{array}{l}\text { Rond wintergroen } \\
\text { Rundblättriges Wintergrün } \\
\text { Klit-Vintergrøn }\end{array}$ & EN & WAT & Vu & EN & CR & * \\
\hline Radiola linoides Roth & $\begin{array}{l}\text { Dwergvlas } \\
\text { Zwerg-Lein } \\
\text { Tusindfrø }\end{array}$ & vu & WAT & VU & EN & EN & * \\
\hline Ranunculus auricomus $\mathrm{L}$. & $\begin{array}{l}\text { Gulden boterbloem } \\
\text { Gold-Hahnenfuß } \\
\text { Nyrebladet Ranunkel }\end{array}$ & su & & su & - & - & - \\
\hline Ranunculus baudotii Godr. & $\begin{array}{l}\text { Zilte waterranonkel } \\
\text { Brackwasser-Wasser- } \\
\text { hahnenfuß } \\
\text { Strand-Vandranunkel }\end{array}$ & Vu & EUT & * & EN & VU & * \\
\hline Ranunculus hederaceus $\mathrm{L}$. & $\begin{array}{l}\text { Klimopwaterranonkel } \\
\text { Efeublättriger Hahnenfuß } \\
\text { Vedbend-Vandranunkel }\end{array}$ & EX? & WAT & EX & - & $\left({ }^{*}\right)$ & - \\
\hline Ranunculus tripartitus DC. & $\begin{array}{l}\text { Driedelige waterranonkel } \\
\text { Dreiteiliger Wasserhahnen- } \\
\text { fuß }\end{array}$ & EX & WAT & EX & - & - & - \\
\hline $\begin{array}{l}\text { Rhinanthus angustifolius } \\
\text { C.C.Gmelin (Coll.) }\end{array}$ & $\begin{array}{l}\text { Grote ratelaar } \\
\text { Großer Klappertopf } \\
\text { Stor Skjaller }\end{array}$ & Vu & WAT,EUT & Vu & VU & Vu & * \\
\hline $\begin{array}{l}\text { Rhinanthus angustifolius ssp. } \\
\text { halophilus (U.Schneider) Soo }\end{array}$ & Strand-Skjaller & VU? & & $(*)$ & - & & Vu \\
\hline Rhinanthus minor $\mathrm{L}$. & $\begin{array}{l}\text { Kleine ratelaar } \\
\text { Kleiner Klappertopf } \\
\text { Liden Skjaller }\end{array}$ & Vu & $A G R$ & $*$ & Vu & vu & * \\
\hline $\begin{array}{l}\text { Rhynchospora alba } \\
\text { (L.) Vahl }\end{array}$ & $\begin{array}{l}\text { Witte snavelbies } \\
\text { Weißes Schnabelried } \\
\text { Hvid Næbfrø }\end{array}$ & CR? & WAT & EX & - & $(*)$ & * \\
\hline
\end{tabular}




\begin{tabular}{|c|c|c|c|c|c|c|c|}
\hline & & \multirow{2}{*}{$\begin{array}{c}\text { Red List } \\
\text { (trilateral) }\end{array}$} & \multirow[t]{2}{*}{ Threats } & \multicolumn{4}{|c|}{$\begin{array}{l}\text { Status of threat in the subre- } \\
\text { gions of the Wadden Sea Area }\end{array}$} \\
\hline & & & & NL & Nds & $\mathrm{SH}$ & DK \\
\hline $\begin{array}{l}\text { Rhynchospora fusca } \\
\text { (L.) Ait. fil. }\end{array}$ & $\begin{array}{l}\text { Bruine snavelbies } \\
\text { Braunes Schnabeiried } \\
\text { Brun Næbfrø }\end{array}$ & CR & WAT & EX & - & EX & * \\
\hline Rosa pimpinellifolia L. & $\begin{array}{l}\text { Duinroosje } \\
\text { Dünen-Rose } \\
\text { Klit-Rose } \\
\end{array}$ & VU & & * & EN & VU & * \\
\hline $\begin{array}{l}\text { Ruppia cirrhosa (Petagna) } \\
\text { Grande }\end{array}$ & $\begin{array}{l}\text { Spiraalruppia } \\
\text { Schraubige Salde } \\
\text { Langstilket Havgræs } \\
\end{array}$ & EN & EUT, WAT & Vu & SU & $C R$ & * \\
\hline Ruppia maritima L. & \begin{tabular}{|l} 
Snavelruppia \\
Meeres-Salde \\
Alm. Havgræs
\end{tabular} & EN & $\begin{array}{l}\text { EUT, HAB, } \\
\text { WAT }\end{array}$ & Vu & EN & CR & * \\
\hline Sagina nodosa (L.) Fenzl & $\begin{array}{l}\text { Sierlijke vetmuur } \\
\text { Knotiges Mastkraut } \\
\text { Knude-Firling }\end{array}$ & vu & WAT & * & EN & VU & * \\
\hline $\begin{array}{l}\text { Sagina subulata (Swartz) } \\
\text { C.Presl. }\end{array}$ & $\begin{array}{l}\text { Priemvetmuur } \\
\text { Pfriemen-Mastkraut } \\
\text { Syl-Firling }\end{array}$ & CR & WAT & - & - & CR & * \\
\hline Sagittaria sagittifolia $\mathrm{L}$. & \begin{tabular}{|l} 
Pijlkruid \\
Pfeilkraut \\
Alm. Pilblad \\
\end{tabular} & * & & su & - & - & * \\
\hline Salix hastata L. & $\begin{array}{l}\text { Spieß-Weide } \\
\text { Spyd-Pil }\end{array}$ & * & WAT & - & - & SU & * \\
\hline Samolus valerandi $\mathrm{L}$. & $\begin{array}{l}\text { Waterpunge } \\
\text { Salz-Bunge } \\
\text { Samel }\end{array}$ & Vu & WAT & * & EN & - & - \\
\hline $\begin{array}{l}\text { Schoenoplectus pungens } \\
\text { (Vahl) Palla }\end{array}$ & $\begin{array}{l}\text { Stekende bies } \\
\text { Amerikanische Teichsimse }\end{array}$ & CR & WAT & $\mathrm{CR}$ & $C R$ & EX & - \\
\hline $\begin{array}{l}\text { Schoenoplectus triqueter } \\
\text { (L.) Palla }\end{array}$ & $\begin{array}{l}\text { Driekantige bies } \\
\text { Dreikantige Teichsimse }\end{array}$ & EN & HAB, WAT & - & EN & $\mathrm{CR}$ & - \\
\hline $\begin{array}{l}\text { Schoenoplectus } X \text { carinatus } \\
\text { Smith }\end{array}$ & $\begin{array}{l}\text { Bastaardbies } \\
\text { Tide-Simse }\end{array}$ & EN & & - & EN & SU & - \\
\hline Schoenus nigricans $\mathrm{L}$. & $\begin{array}{l}\text { Knopbies } \\
\text { Schwarzes Kopfried } \\
\text { Sort Skæne }\end{array}$ & EN & WAT & vu & EN & EX & - \\
\hline Scleranthus perennis L. & $\begin{array}{l}\text { Overblijvende hardbloem } \\
\text { Ausdauernder Knäuel } \\
\text { Flerärig Knavel }\end{array}$ & Vu & & * & VU & Vu & - \\
\hline
\end{tabular}




\begin{tabular}{|c|c|c|c|c|c|c|c|}
\hline & & \multirow{2}{*}{$\begin{array}{c}\text { Red List } \\
\text { (trilateral) }\end{array}$} & \multirow[t]{2}{*}{ Threats } & \multicolumn{4}{|c|}{$\begin{array}{l}\text { Status of threat in the subre- } \\
\text { gions of the Wadden Sea Area }\end{array}$} \\
\hline & & & & NL & Nds & $\mathrm{SH}$ & DK \\
\hline Scorzonera humilis $\mathrm{L}$. & $\begin{array}{l}\text { Kleine schorseneer } \\
\text { Niedrige Schwarzwurzel } \\
\text { Lav Skorsoner }\end{array}$ & EN & EUT, AGR & - & - & EN & - \\
\hline Scutellaria minor Huds. & $\begin{array}{l}\text { Klein glidkruid } \\
\text { Kleines Heimkraut } \\
\text { Liden Skjolddrager }\end{array}$ & EN & WAT, EUT & EN & - & - & - \\
\hline Sedum album L. & $\begin{array}{l}\text { Wit vetkruid } \\
\text { Weiße Fetthenne } \\
\text { Hvid Stenurt }\end{array}$ & SU & & su & - & - & - \\
\hline Senecio erucifolius L. & $\begin{array}{l}\text { Viltig kruiskruid } \\
\text { Raukenblättriges Greis- } \\
\text { kraut } \\
\text { Smalfliget Brandbæger }\end{array}$ & EN & WAT & EX & - & * & - \\
\hline Silene nutans $\mathrm{L}$. & $\begin{array}{l}\text { Nachtsilene } \\
\text { Nickendes Leimkraut } \\
\text { Nikkende Limurt }\end{array}$ & CR & $\begin{array}{l}\text { HAB, AGR, } \\
\text { EUT }\end{array}$ & - & - & $C R$ & - \\
\hline Silene otites (L.) Wibel & $\begin{array}{l}\text { Oorsilene } \\
\text { Ohrlöffel-Leimkraut } \\
\text { Klit-Limurt }\end{array}$ & vu & HAB, AGR & Vu & VU & $\mathrm{CR}$ & SU \\
\hline Smyrnium olusatrum L. & $\begin{array}{l}\text { Zwartmoeskervel } \\
\text { Makedonisk Persille }\end{array}$ & su & & SU & - & - & - \\
\hline Sparganium natans $\mathrm{L}$. & $\begin{array}{l}\text { Kleinste egelskop } \\
\text { Spæd Pindsvineknop }\end{array}$ & CR & WAT & EX & - & - & * \\
\hline Spergula morisonii Boreau & $\begin{array}{l}\text { Heidespurrie } \\
\text { Frühlings-Spergel } \\
\text { Văr-Spergel }\end{array}$ & su & & su & - & - & - \\
\hline Stachys arvensis (L.) L. & $\begin{array}{l}\text { Akkerandoorn } \\
\text { Acker-Ziest } \\
\text { Ager-Galtetand }\end{array}$ & EN? & AGR & CR & EN & $\left({ }^{*}\right)$ & - \\
\hline Stellaria aquatica (L.) Scop. & $\begin{array}{l}\text { Watermuur } \\
\text { Wasserdarm } \\
\text { Kiøvkrone } \\
\end{array}$ & CR & & SU & - & - & EX \\
\hline Subularia aquatica L. & \begin{tabular}{|l} 
Priemkruid \\
Pfriemenkresse \\
Sylblad
\end{tabular} & EX & & - & - & EX & - \\
\hline Taraxacum celticum agg. & $\begin{array}{l}\text { Schraallandpaardebloem } \\
\text { Eng-Malkebøtte }\end{array}$ & vu & AGR, WAT & VU & vu & - & $?$ \\
\hline Taraxacum palustre agg. & $\begin{array}{l}\text { Moeraspaardebloem } \\
\text { Sumpf-Kuhblume } \\
\text { Smalbladet Malkebøtte }\end{array}$ & Vu & WAT, AGR & Vu & - & - & - \\
\hline
\end{tabular}




\begin{tabular}{|c|c|c|c|c|c|c|c|}
\hline & & \multirow{2}{*}{$\begin{array}{c}\text { Red List } \\
\text { (trilateral) }\end{array}$} & \multirow[t]{2}{*}{ Threats } & \multicolumn{4}{|c|}{$\begin{array}{l}\text { Status of threat in the subre- } \\
\text { gions of the Wadden Sea Area }\end{array}$} \\
\hline & & & & NL & Nds & $\mathrm{SH}$ & DK \\
\hline Thalictrum flavum L. & $\begin{array}{l}\text { Poelruit } \\
\text { Gelbe Wiesenraute } \\
\text { Gul-Frøstjerne }\end{array}$ & ĆR? & & $\left({ }^{*}\right)$ & - & $\mathrm{CR}$ & * \\
\hline Thalictrum minus $\mathrm{L}$. & $\begin{array}{l}\text { Kleine ruit } \\
\text { Kleine Wiesenraute } \\
\text { Sand-Frøstjerne }\end{array}$ & vu & & * & VU & EX & * \\
\hline Thymus pulegioides $\mathrm{L}$. & $\begin{array}{l}\text { Grote tijm } \\
\text { Gemeiner Thymian } \\
\text { Bredbladet Timian }\end{array}$ & su & & SU & - & - & - \\
\hline Torilis nodosa (L.) Gaertn. & $\begin{array}{l}\text { Knopig doornzaad } \\
\text { Knäuel-Klettenkerbel } \\
\text { Knudret Randfrø }\end{array}$ & vu & AGR & Vu & EN & VU & - \\
\hline $\begin{array}{l}\text { Trichophorum cespitosum } \\
\text { (L.) Hartman }\end{array}$ & $\begin{array}{l}\text { Veenbies } \\
\text { Rasige Haarsimse } \\
\text { Tue Kogleaks }\end{array}$ & CR & WAT & - & EX & EN & * \\
\hline Trientalis europaeus $\mathrm{L}$. & $\begin{array}{l}\text { Zevenster } \\
\text { Europaischer Siebenstern } \\
\text { Skovstjerne }\end{array}$ & * & & * & - & su & - \\
\hline Trifolium ornithopodioides $\mathrm{L}$. & $\begin{array}{l}\text { Vogelpootklaver } \\
\text { Vogelfußartiger Klee }\end{array}$ & * & & * & - & su & \\
\hline Trifolium striatum $\mathrm{L}$. & $\begin{array}{l}\text { Gestreepte klaver } \\
\text { Streifen-Klee } \\
\text { Stribet Kløver }\end{array}$ & su & & SU & - & SU & - \\
\hline Triglochin palustre $\mathrm{L}$. & $\begin{array}{l}\text { Moeraszoutgras } \\
\text { Sumpf-Dreizack } \\
\text { Kær-Trehage }\end{array}$ & vu & AGR & * & EN & Vu & * \\
\hline Tuberaria guttata (L.) Fourr. & $\begin{array}{l}\text { Gevlekt zonneroosje } \\
\text { Geflecktes Sandröschen } \\
\text { Enårig Stensoløje }\end{array}$ & CR & $?$ & CR & $C R$ & - & - \\
\hline Ulex europaeus L..' & $\begin{array}{l}\text { Gaspeldoorn } \\
\text { Stechginster } \\
\text { Tornblad } \\
\end{array}$ & EN & HAB & Vu & EN & EN & $\left({ }^{\star}\right)$ \\
\hline Utricularia australis R.Br. & $\begin{array}{l}\text { Loos blaasjeskruid } \\
\text { Südlicher Wasserschlauch } \\
\text { Slank Blærerod } \\
\end{array}$ & VU & WAT, EUT & vu & VU & - & * \\
\hline Utricularia minor $\mathrm{L}$. & $\begin{array}{l}\text { Klein blaasjeskruid } \\
\text { Kleiner Wasserschlauch } \\
\text { Liden Blærerod }\end{array}$ & CR & WAT & EX & - & - & * \\
\hline Vaccinium macrocarpon Ait. & $\begin{array}{l}\text { Grote veenbes } \\
\text { Großfrüchtige Moosbeere } \\
\text { Storfrugtet Tranebær }\end{array}$ & * & & * & - & su & - \\
\hline
\end{tabular}




\begin{tabular}{|c|c|c|c|c|c|c|c|}
\hline & & \multirow{2}{*}{$\begin{array}{l}\text { Red List } \\
\text { (trilaterai) }\end{array}$} & \multirow[t]{2}{*}{ Threats } & \multicolumn{4}{|c|}{$\begin{array}{l}\text { Status of threat in the subre- } \\
\text { gions of the Wadden Sea Area }\end{array}$} \\
\hline & & & & $\mathrm{NL}$ & Nds & $\mathrm{SH}$ & DK \\
\hline Vaccinium myrtillus $\mathrm{L}$. & $\begin{array}{l}\text { Blauwe bosbes } \\
\text { Heidelbeere } \\
\text { Blábær }\end{array}$ & vu & & su & * & CR & - \\
\hline Vaccinium vitis-idaea $\mathrm{L}$. & $\begin{array}{l}\text { Rode bosbes } \\
\text { Preiselbeere } \\
\text { Alm. Tyttebær }\end{array}$ & EN & & * & * & $C R$ & - \\
\hline $\begin{array}{l}\text { Viola tricolor ssp. curtisii } \\
\text { (Forst.) Syme }\end{array}$ & $\begin{array}{l}\text { Duinviooltje } \\
\text { Dünen-Stiefmütterchen } \\
\text { Klit-Stedmoderblomst }\end{array}$ & * & DIS, HAB & * & * & VU & * \\
\hline Vulpia bromoides (L.) S.F.Gray & $\begin{array}{l}\text { Eekhoorngras } \\
\text { Trespen-Federschwingel } \\
\text { Langstakket Væselhale } \\
\end{array}$ & EN & AGR, EUT & $\mathrm{VU}$ & $C R$ & CR & - \\
\hline $\begin{array}{l}\text { Xanthium albinum ssp. albi- } \\
\text { num (Widder) H. Schoiz s.str. }\end{array}$ & $\begin{array}{l}\text { Oeverstekelnoot } \\
\text { Elb-Spitzklette }\end{array}$ & su & & - & - & SU & - \\
\hline $\begin{array}{l}\text { Zannichellia palustris L. Ssp. } \\
\text { polycarpa (Rchb) K. Richter }\end{array}$ & $\begin{array}{l}\text { Brede zannichellia } \\
\text { Sumpf-Teichfaden } \\
\text { Stor Vandkraus } \\
\end{array}$ & EX & WAT & $E X$ & - & - & - \\
\hline Zostera marina L. & $\begin{array}{l}\text { Groot zeegras } \\
\text { Gewöhnliches Seegras } \\
\text { Alm. Bændeltang } \\
\end{array}$ & vu & $\begin{array}{l}\text { EXL, EUT, } \\
\text { PAR }\end{array}$ & EN & EN & Vu & * \\
\hline Zostera noltii Homem. & $\begin{array}{l}\text { Klein zeegras } \\
\text { Zwerg-Seegras } \\
\text { Dværg-Bændeltang }\end{array}$ & vu & EXL, EUT & SU & EN & Vu & * \\
\hline
\end{tabular}

Introduced in Denmark.

2 Anthropochorous in Denmark.

3 Anthropochorous in the Wadden Sea area.

Status of threat:

EX $=$ Extinct $; \mathbf{C R}=$ Critical $_{;}$EN $=$Endangered $; \mathbf{V U}=$ Vulnerable $; \mathbf{S U}=$ Susceptible $^{*}$ " = not endan . gered; ? = status of endangerment is not exactly known $_{i}=$ species does not occur $\boldsymbol{r}_{i}-(?)=$ species probably does not occur; $(")=$ it is unknown if or if not this species occurs.

Threats:

$\mathrm{AFF}=$ afforestation $; \mathrm{AGR}=$ agriculture $; \mathrm{CLI}=$ climatic change DIS $=$ disturbance $; \mathrm{EUT}=$ eutrophi cation; $\mathrm{EXL}=$ exploitation of living resources $; \mathrm{EXM}=$ mineral exploitation; $\mathrm{FOR}=$ forestry; $\mathrm{HAB}=$ loss of habitat; $\mathrm{PAR}=$ parasites $; \mathrm{POL}=$ pollution; $\mathrm{WAT}=$ water regulation .

For more detailed descriptions see the general introduction to the Red Lists. 